\title{
Regularity of Geometric quadratic stochastic operator generated by 2-partition of infinite points
}

\author{
Siti Nurlaili Karim, Nur Zatul Akmar Hamzah, Nasir Ganikhodjaev \\ Department of Computational and Theoretical Sciences, Kulliyyah of Science, International Islamic University Malaysia, Jalan Sultan Ahmad Shah, \\ Bandar Indera Mahkota, 25200 Kuantan, Pahang, Malaysia
}

* Corresponding author: zatulakmar@iium.edu.my

Article history

Received 29 August 2019

Revised 15 January 2020

Accepted 28 January 2020

Published Online 15 June 2020

\begin{abstract}
In this research, we construct a class of quadratic stochastic operator called Geometric quadratic stochastic operator generated by arbitrary 2-partition $\xi$ of infinite points on a countable state space $X$, where $X=\{0,1,2, \ldots\}$. We also study the limiting behavior of such operator by proving the existence of the limit of the sequence $V^{n} \mu$ through the convergence of the trajectory to a unique fixed point. It is established that such operator is a regular transformation.
\end{abstract}

Keywords: Infinite points, countable state space, quadratic stochastic operator, regular transformation

(C) 2020 Penerbit UTM Press. All rights reserved

\section{INTRODUCTION}

It is widely recognized that the theory of quadratic stochastic operator frequently arises due to its enormous contribution as a source of analysis for the investigation of dynamical properties and modeling in diverse domains.

A quadratic stochastic operator (qso) is also known as an evolutionary operator since it is used to present the time evolution of differing species in biology. Let us shortly mention on how such kind of operators arise in population genetics. We consider biological population, that is, a community of organisms that closed with respect to reproduction. Assume that every individual in this population belongs to one of the species $1,2, \ldots, m$. We denote the probability that the species of parents $i$ and $j$ interbreed to produce species $k$ via $P_{i j, k}$ and $x^{(0)}=\left(x_{1}{ }^{(0)}, \ldots, x_{m}{ }^{(0)}\right)$ be a probability distribution of species at an initial state. Hence, $x_{k}^{(1)}=\sum_{i, j=1}^{m} P_{i j, k} x_{i}^{(0)} x_{j}^{(0)}$ is the total probability of the species in the first generation of direct descendants.

The idea of qso was initiated by Bernstein in 1924. Since then, a lot of papers are devoted to study such operators (Ganikhodjaev, 1993, 1994; Kesten, 1970; Lyubich, 1978, 1992). Note that one of the main problems in the nonlinear operator theory is to study the asymptotical behaviour of nonlinear operators. Even in the class of quadratic stochastic operator which is the simplest nonlinear operators, the problem remains open. The difficulty of the problem in qso depends on the given cubic matrix $\left(P_{i j k}\right)_{i, j, k=1}^{m}$, where $m$ is the dimensional simplex. In order to study this problem, many researchers are devoted to construct several classes of qso. They describe and study their properties, then investigate their trajectory behaviour.

Generally, the study of qso can be divided into two main cases, i.e. Volterra and non-Volterra quadratic stochastic operators. The idea of Volterra quadratic stochastic operators can be reinterpreted in terms of its biological view in which the offspring repeats one of its parents. The trajectory behavior of such operators has been studied in many previous works (Ganikhodjaev \& Hamzah, 2015c; Ganikhodzhaev, Mukhamedov, \& Rozikov, 2011; Kesten, 1970; Losert \& Akin, 1983; Lyubich, 1978, 1992; Mukhamedov, 2000; Zakharevich, 1978).

In the case of non-Volterra quadratic stochastic operators, the study of such operators is still an open problem where new classes of quadratic stochastic operator are continuously constructed and further investigated in many papers. In Ganikhodjaev \& Hamzah (2014c, 2014a, 2014b, 2015a), the authors introduced and studied Poisson and Geometric qso on countable state space and Gaussian qso on continual state space. Another class of qso which called as Lebesgue qso on the segment $[0,1]$ generated by 2-partition $\xi$ is introduced and studied by Ganikhodjaev \& Hamzah (2015b).

In this paper, we are motivated to introduce another class of quadratic stochastic operator called Geometric quadratic stochastic operator generated by 2-partition $\xi$ of infinite points defined on countable state space. In the next section, we provide the concept of quadratic stochastic operator on the set of all probability measures and the definition of Geometric quadratic stochastic operator in details.

\section{PRELIMINARIES}

Let $(X, F)$ be a measurable space, where $X$ is a state space, $F$ is a $\sigma$-algebra on $X$, and $S(X, F)$ be a set of all probability measures on a measurable space $(X, F)$. We consider a family of functions $\quad\{P(x, y, A): x, y \in X, A \in F\} \quad$ on $\quad X \times X \times F \quad$ where $P(x, y, A) \in S(X, F)$ for fixed $A$ is regarded as a measurable function of two variables and $P(x, y, A)=P(y, x, A)$ for any $x, y \in X$ and $A \in F$. 
A nonlinear transformation called quadratic stochastic operator (qso) $V: S(X, F) \rightarrow S(X, F)$ is defined by

$$
(V \mu)(A)=\int_{X} \int_{X} P(x, y, A) d \mu(x) d \mu(y),
$$

where $\mu \in S(X, F)$ and $A \in F$.

Note that in this paper, we consider a countable set $X$ where $X={ }_{+}$is the set of all nonnegative integers. On ${ }_{+}$state space, one can consider a discrete probability distribution, i.e. Poisson distribution, Geometric distribution, etc. Throughout this paper, we consider a Geometric distribution which has the following form

$$
G_{r}(k)=(1-r) r^{k},
$$

for any $k \in+$

Since we consider the case where the state space $X={ }_{+}$, then we can define a measure $\{k\}$ as $P(i, j,\{k\})$ and by additivity, we have

$$
P(i, j, A)=\sum_{k \in A} P(i, j,\{k\}),
$$

where we denote $P(i, j,\{k\})$ as $P_{i j, k}$.

We define a family of functions $\left\{P_{i j, k}: i, j, k \in{ }_{+}\right\}$which satisfies the following conditions:

i. $\quad P_{i j, v} \in S\left({ }_{+}, F\right)$ is the probability measure,

ii. $\quad P_{i j, k}=P_{j i, k}$

Hence, a qso (1) can be written as

$$
V \mu(k)=\sum_{i=0}^{\infty} \sum_{j=0}^{\infty} P_{i j, k} \mu(i) \mu(j),
$$

where $k \in{ }_{+}$for a measure $\mu \in S\left({ }_{+}, F\right)$.

Definition 1: A qso $V$ (2) is called as a Geometric quadratic stochastic operator, if for any $i, j \in X$, the probability measure $P(i, j, \cdot)$ is the Geometric distribution $G_{r(i, j)}$ with a real parameter $r(i, j)=r(j, i), 0<r(i, j)<1$.

Assume that $\left\{V^{n}(\mu) \in S(X, F): n=0,1,2, \ldots\right\}$ is a trajectory of the initial measure $\mu \in S(X, F)$, where $V^{n+1}(\mu)=V\left(V^{n}(\mu)\right)$ for all $n=0,1,2, \ldots$ with $V^{0}(\mu)=\mu$. The definitions of fixed point and regularity of qso $V$ are presented in the following.

Definition 2: A point $\mu \in S(X, F)$ is called a fixed point of a qso $V$, if $V(\mu)=\mu$.

Let $\operatorname{Fix}(V)$ be a set of all fixed points of qso $V$.

Definition 3: A qso $V$ is called a regular if for any initial point $\mu \in S(X, F)$, the limit

$$
\lim _{n \rightarrow \infty} V^{n}(\mu)
$$

exists.

Limit behaviour of the trajectories and the fixed points of the qso have been studied in many publications (Akin \& Losert, 1984; Akin, 1993; Bernstein, 1924; Ganikhodjaev, Ganikhodjaev, \& Jamilov, 2015; Ganikhodjaev, 1993, 1994; Ganikhodzhaev \& Zanin, 2004; Ganikhodzhaev et al., 2011; Hofbauer \& Sigmund, 1998; Jenks, 1969;
Kesten, 1970; Lyubich, 1978, 1992; Mukhamedov \& Embong, 2015; Ulam, 1960; Volterra, 1931; Zakharevich, 1978).

Suppose that $\xi=\left\{A_{1}, A_{2}\right\}$ be a measurable 2-partition of the state space ${ }_{+}$, where $A_{1} \subset{ }_{+}, A_{2}={ }_{+} \backslash A_{1}$, and $\varsigma=\left\{B_{1}, B_{2}\right\}$ with $B_{1}=\left(A_{1} \times A_{1}\right) \cup\left(A_{2} \times A_{2}\right)$ and $B_{2}=\left(A_{1} \times A_{2}\right) \cup\left(A_{2} \times A_{1}\right)$. Then, we define the family $\left\{P_{i j, k}: i, j, k \in+\right\}$ as follows:

$$
P_{i j, k}= \begin{cases}\left(1-r_{1}\right) r_{1}^{k} & \text { if }(i, j) \in B_{1}, \\ \left(1-r_{2}\right) r_{2}^{k} & \text { if }(i, j) \in B_{2},\end{cases}
$$

where $r_{1}, r_{2} \in r(i, j)$ is a real parameter.

The study of Geometric qso was introduced and studied by Ganikhodjaev \& Hamzah (2015a) where it has been proven that such operator generated by 1-partition was a regular transformation with up to three parameters. Motivated by the study by Ganikhodjaev \& Hamzah (2015b, 2015a), the authors in Karim, Hamzah, \& Ganikhodjaev (2019) constructed and investigated a Geometric quadratic stochastic operator generated by 2-partition of singleton.

In this present paper, we intend to study a Geometric quadratic stochastic operator generated by 2-partition of infinite points by considering $A_{1}$ as a set of all nonnegative integers divisible by 3 and following the case where $A_{1}$ consists of arbitrary infinite points.

\section{GEOMETRIC QUADRATIC STOCHASTIC OPERATOR GENERATED BY 2-PARTITION $\xi$ OF MODULUS 3}

Let $A_{1}=3+$ is a set of infinite points with modulus 3 to generate the 2-partition $\xi$ while $A_{2}=+\backslash A_{1}$. Now, we consider a Geometric quadratic stochastic operator such that as defined in (4). For any measure $\mu \in S\left({ }_{+}, F\right)$, consider that $A(\mu)=\sum_{i=0}^{\infty} \mu(3 i) \quad$ and $B(\mu)=\sum_{i=0}^{\infty} \mu(3 i+1)+\sum_{i=0}^{\infty} \mu(3 i+2)=1-\sum_{i=0}^{\infty} \mu(3 i), \quad$ where $A(\mu)+B(\mu)=1$. We can easily verify for Geometric distribution $G_{r}$, $A\left(G_{r}\right)=\frac{1}{r^{2}+r+1}$ and $B\left(G_{r}\right)=\frac{r^{2}+r}{r^{2}+r+1}$. Thus, we have $V \mu(k)=\sum_{i=0}^{\infty} \sum_{j=0}^{\infty} P_{i j, k} \mu(i) \mu(j)$

$=\sum_{i, j=0}^{\infty} P_{3 i, 3 j, k} \mu(3 i) \mu(3 j)+\sum_{i, j=0}^{\infty} P_{3 i+1,3 j+1, k} \mu(3 i+1) \mu(3 j+1)$

$+\sum_{i, j=0}^{\infty} P_{3 i+2,3 j+2, k} \mu(3 i+2) \mu(3 j+2)+\sum_{i, j=0}^{\infty} P_{3 i+1,3 j+2, k} \mu(3 i+1) \mu(3 j+2)$

$+\sum_{i, j=0}^{\infty} P_{3 i+2,3 j+1, k} \mu(3 i+2) \mu(3 j+1)+\sum_{i, j=0}^{\infty} P_{3 i, 3 j+1, k} \mu(3 i) \mu(3 j+1)$

$+\sum_{i, j=0}^{\infty} P_{3 i+1,3 j, k} \mu(3 i+1) \mu(3 j)+\sum_{i, j=0}^{\infty} P_{3 i, 3 j+2, k} \mu(3 i) \mu(3 j+2)$

$+\sum_{i, j=0}^{\infty} P_{3 i+2,3 j, k} \mu(3 i+2) \mu(3 j)$

$=\left(1-r_{1}\right) r_{1}^{k}\left[A^{2}(\mu)+B^{2}(\mu)\right]+\left(1-r_{2}\right) r_{2}^{k}[2 A(\mu) B(\mu)]$, and

$V^{2} \mu(k)=\sum_{i=0}^{\infty} \sum_{j=0}^{\infty} P_{i j, k} V \mu(i) V \mu(j)$

$=\sum_{i, j=0}^{\infty} P_{3 i, 3 j, k} V \mu(3 i) V \mu(3 j)+\sum_{i, j=0}^{\infty} P_{3 i+1,3 j+1, k} V \mu(3 i+1) V \mu(3 j+1)$ 
$+\sum_{i, j=0}^{\infty} P_{3 i+2,3 j+2, k} V \mu(3 i+2) V \mu(3 j+2)+\sum_{i, j=0}^{\infty} P_{3 i+1,3 j+2, k} V \mu(3 i+1) V \mu(3 j+2)$

$+\sum_{i, j=0}^{\infty} P_{3 i+2,3 j+1, k} V \mu(3 i+2) V \mu(3 j+1)+\sum_{i, j=0}^{\infty} P_{3 i, 3 j+1, k} V \mu(3 i) V \mu(3 j+1)$

$+\sum_{i, j=0}^{\infty} P_{3 i+1,3 j, k} V \mu(3 i+1) V \mu(3 j)+\sum_{i, j=0}^{\infty} P_{3 i, 3 j+2, k} V \mu(3 i) V \mu(3 j+2)$

$+\sum_{i, j=0}^{\infty} P_{3 i+2,3 j, k} V \mu(3 i+2) V \mu(3 j)$

$=\left(1-r_{1}\right) r_{1}^{k}\left[A^{2}(V \mu)+B^{2}(V \mu)\right]+\left(1-r_{2}\right) r_{2}^{k}[2 A(V \mu) B(V \mu)]$.

Next, by simple calculations, it is given that

$$
\begin{aligned}
& A(V \mu)=\frac{1}{r_{1}^{2}+r_{1}+1}\left[A^{2}(\mu)+B^{2}(\mu)\right] \\
& +\frac{1}{r_{2}^{2}+r_{2}+1}[2 A(\mu) B(\mu)], \text { and } \\
& B(V \mu)=\frac{r_{1}^{2}+r_{1}}{r_{1}^{2}+r_{1}+1}\left[A^{2}(\mu)+B^{2}(\mu)\right] \\
& +\frac{r_{2}^{2}+r_{2}}{r_{2}^{2}+r_{2}+1}[2 A(\mu) B(\mu)] .
\end{aligned}
$$

Simply by using mathematical induction on the sequence $V^{n} \mu(k)$, we attain the following

$$
\begin{aligned}
V^{n+1} \mu(k) & =\left(1-r_{1}\right) r_{1}^{k}\left[A^{2}\left(V^{n} \mu\right)+B^{2}\left(V^{n} \mu\right)\right] \\
& +\left(1-r_{2}\right) r_{2}^{k}\left[2 A\left(V^{n} \mu\right) B\left(V^{n} \mu\right)\right],
\end{aligned}
$$

for $n=0,1,2, \ldots$. Moreover, for parameters $A\left(V^{n} \mu\right)$ and $B\left(V^{n} \mu\right)$, they follow that

$$
\begin{aligned}
& A\left(V^{n+1} \mu\right)=\frac{1}{r_{1}^{2}+r_{1}+1}\left[A^{2}\left(V^{n} \mu\right)+B^{2}\left(V^{n} \mu\right)\right] \\
& +\frac{1}{r_{2}^{2}+r_{2}+1}\left[2 A\left(V^{n} \mu\right) B\left(V^{n} \mu\right)\right], \text { and } \\
& B\left(V^{n+1} \mu\right)=\frac{r_{1}^{2}+r_{1}}{r_{1}^{2}+r_{1}+1}\left[A^{2}\left(V^{n} \mu\right)+B^{2}\left(V^{n} \mu\right)\right] \\
& +\frac{r_{2}^{2}+r_{2}}{r_{2}^{2}+r_{2}+1}\left[2 A\left(V^{n} \mu\right) B\left(V^{n} \mu\right)\right] .
\end{aligned}
$$

Notice that the limit behaviour of the recurrent equation (6) is bounded by the limit behaviour of recurrent equations (7).

We have $A\left(V^{n} \mu\right)+B\left(V^{n} \mu\right)=1 \quad$ where $\quad A\left(V^{n} \mu\right) \geq 0 \quad$ and $B\left(V^{n} \mu\right) \geq 0$. Hence, as $n \rightarrow \infty$ the recurrent equations (7) can be rewritten as follows:

$$
\begin{aligned}
& x=A\left(G_{r_{1}}\right) \cdot\left(x^{2}+y^{2}\right)+2 A\left(G_{r_{2}}\right) \cdot(x y), \text { and } \\
& y=B\left(G_{r_{1}}\right) \cdot\left(x^{2}+y^{2}\right)+2 B\left(G_{r_{2}}\right) \cdot(x y),
\end{aligned}
$$

where $A\left(V^{n} \mu\right)$ and $B\left(V^{n} \mu\right)$ are represented as variable $x$ and $y$ respectively with $x \geq 0, y \geq 0$, and $x+y=1$.

It is sufficient to solve for the first equation in (8). Hence, we may rewrite the mentioned equation as follows:

$$
x=A\left(G_{r_{1}}\right)\left[x^{2}+(1-x)^{2}\right]+2 A\left(G_{r_{2}}\right)[x(1-x)] .
$$

Obviously, the equation (9) is a quadratic equation. We may rewrite the right-hand side equation as follows:

$$
g(x)=2\left[A\left(G_{r_{1}}\right)-A\left(G_{r_{2}}\right)\right] x^{2}-2\left[A\left(G_{r_{1}}\right)-A\left(G_{r_{2}}\right)\right] x+A\left(G_{r_{1}}\right),
$$

where $0<r_{1}, r_{2}<1$. Then, the equation (10) can be regarded as a function, where

$$
g(0)=g(1)=A\left(G_{r_{1}}\right) .
$$

This implies that such function maps the segment $[0,1]$ into itself. Therefore, the following statements are established.

Theorem 1: The transformation (10) has a unique fixed point which belongs to the open interval $(0,1)$.

Proof Recall that a quadratic equation has at most two roots. In fact, it can be easily shown that the equation

$$
\begin{aligned}
x & =2\left[A\left(G_{r_{1}}\right)-A\left(G_{r_{2}}\right)\right] x^{2}-2\left[A\left(G_{r_{1}}\right)-A\left(G_{r_{2}}\right)\right] x+A\left(G_{r_{1}}\right) \\
& =2(a-b) x^{2}-2(a-b) x+a,
\end{aligned}
$$

where $a=A\left(G_{r_{1}}\right)$ and $b=A\left(G_{r_{2}}\right)$ has a root in the interval $(-\infty, 0)$ and $(1, \infty)$ when $2(a-b)<0$ and $2(a-b)>0$, respectively. Another case to be considered is when $2(a-b)=0$, where the equation becomes a linear with $a>0$. Hence, for all considered cases, a root in $[0,1]$ is unique. Evidently, this root varies from 0 to 1 . This completes the proof.

Next, we investigate the local character of the fixed point. Let us consider the discriminant of the quadratic equation (11). Hence, we have the following,

$$
\Delta=4(1-a) a+(1-2 b)^{2}
$$

By simple calculations, it is notable that we have $0<\Delta<2$. Now, it remains to show that the function converges to a fixed point to describe the regularity of such operator, whence the following theorem is given.

Theorem 2: A fixed point of the transformation (10) is attractive, if $0<\Delta<2$.

Proof Let $\zeta$ be a fixed point of the quadratic equation (11), where

$$
\zeta=\frac{2(a-b)+1-\sqrt{\Delta}}{4(a-b)}
$$

Suppose that $\lambda=f^{\prime}(\zeta)$, where $f(x)$ is a right-hand side of the equation (11) and its derivative, $f^{\prime}(x)$ is continuous. Then, one can obtain

$$
\lambda=4(a-b) \zeta-2(a-b)
$$

By substituting (13) into (14),

$$
\lambda=1-\sqrt{\Delta} .
$$

It is understood that if $|\lambda|<1$, then $\zeta$ is an attractive point, meanwhile if $|\lambda|>1$, then $\zeta$ is a repelling point. Since $0<\Delta<2$, then we have $1-\sqrt{2}<\lambda<1$ which implies that any unique fixed point in the open interval $(0,1)$ is attractive and the statement of the Theorem 2 follows from the equality (14).

It is shown that the trajectory behavior of qso (6) converges to a unique fixed point in the open interval $(0,1)$. By Definition 3, we say that the limit exists if the trajectory converges. Hence, it is a regular transformation. 
In the succeeding section, we will present the generalization for the Geometric quadratic stochastic operator generated by 2-partition of arbitrary infinite points.

\section{REGULARITY OF GEOMETRIC QUADRATIC STOCHASTIC OPERATOR GENERATED BY 2-PARTITION $\xi$ OF INFINITE POINTS}

To generate 2-partition $\xi$ of arbitrary infinite points, assume that $A_{1}=\left\{x_{1}, \ldots, x_{m}, x_{m+1}, \ldots\right\}$, then we have $A_{2}=+\backslash A_{1}$. Let $A(\mu)=\sum_{p \in A_{1}} \mu(p)$ and $B(\mu)=\sum_{p \in A_{2}} \mu(p)$ where $A(\mu)+B(\mu)=1$. It is easily verified that $A\left(G_{r}\right)=\sum(1-r) r^{p} \quad$ and $B\left(G_{r}\right)=1-\sum_{p \in A_{1}}(1-r) r^{p}$. For any $\mu \in S\left({ }_{+}, F\right)$, we consider the family of functions (4), then we have the qso as follows:

$$
\begin{aligned}
V \mu(k) & =\sum_{i=0}^{\infty} \sum_{j=0}^{\infty} P_{i j, k} \mu(i) \mu(j) \\
& =\sum_{i, j \in A_{1}} P_{i j, k} \mu(i) \mu(j)+\sum_{i, j \in A_{2}} P_{i j, k} \mu(i) \mu(j) \\
& +\sum_{i \in A_{1}} \sum_{j \in A_{2}} P_{i j, k} \mu(i) \mu(j) \sum_{i \in A_{2}} \sum_{j \in A_{1}} P_{i j, k} \mu(i) \mu(j) \\
= & \left(1-r_{1}\right) r_{1}^{k}\left[\sum_{i, j \in A_{1}} \mu(i) \mu(j)+\sum_{i, j \in A_{2}} \mu(i) \mu(j)\right] \\
& +\left(1-r_{2}\right) r_{2}{ }^{k}\left[\sum_{i \in A_{1}} \sum_{j \in A_{2}} \mu(i) \mu(j)+\sum_{i \in A_{2}} \sum_{j \in A_{2}} \mu(i) \mu(j)\right] \\
= & \left(1-r_{1}\right) r_{1}^{k}\left[A^{2}(\mu)+B^{2}(\mu)\right]+\left(1-r_{2}\right) r_{2}^{k}[2 A(\mu) B(\mu)], \text { and } \\
V^{2} \mu(k) & =\sum_{i=0}^{\infty} \sum_{j=0}^{\infty} P_{i j, k} V \mu(i) V \mu(j) \\
& =\sum_{i, j \in A_{1}} P_{i j, k} V \mu(i) V \mu(j)+\sum_{i, j \in A_{2}} P_{i j, k} V \mu(i) V \mu(j) \\
& +\sum_{i \in A_{1}} \sum_{j \in A_{2}} P_{i j, k} V \mu(i) V \mu(j)+\sum_{i \in A_{2}} \sum_{j \in A_{1}} P_{i j, k} V \mu(i) V \mu(j) \\
& =\left(1-r_{1}\right) r_{1}^{k}\left[\sum_{i, j \in A_{1}} V \mu(i) V \mu(j)+\sum_{i, j \in A_{2}} V \mu(i) V \mu(j)\right] \\
& +\left(1-r_{2}\right) r_{2}^{k}\left[\sum_{i \in A_{1}} \sum_{j \in A_{2}} V \mu(i) V \mu(j)+\sum_{i \in A_{2}} \sum_{j \in A_{2}} V \mu(i) V \mu(j)\right] \\
& =\left(1-r_{1}\right) r_{1}^{k}\left[A^{2}(V \mu)+B^{2}(V \mu)\right] \\
& +\left(1-r_{2}\right) r_{2}^{k}[2 A(V \mu) B(V \mu)] .
\end{aligned}
$$

Next, by simple calculations, it is given that

$$
\begin{aligned}
& A(V \mu)=\sum_{p \in A_{1}}\left\{\begin{array}{l}
\left(1-r_{1}\right) r_{1}^{p}\left[A^{2}(\mu)+B^{2}(\mu)\right] \\
+\left(1-r_{2}\right) r_{2}^{p}[2 A(\mu) B(\mu)]
\end{array}\right\}, \text { and } \\
& B(V \mu)=\sum_{p \in A_{1}}\left\{\begin{array}{l}
{\left[1-\left(1-r_{1}\right) r_{1}^{p}\right]\left[A^{2}(\mu)+B^{2}(\mu)\right]} \\
+\left[1-\left(1-r_{2}\right) r_{2}^{p}\right][2 A(\mu) B(\mu)]
\end{array}\right\} .
\end{aligned}
$$

Then, by using mathematical induction on the sequence $V^{n} \mu(k)$, the following recurrent equations are attained

$$
\begin{aligned}
V^{n+1} \mu(k) & =\left(1-r_{1}\right) r_{1}^{k}\left[A^{2}\left(V^{n} \mu\right)+B^{2}\left(V^{n} \mu\right)\right] \\
& +\left(1-r_{2}\right) r_{2}^{k}\left[2 A\left(V^{n} \mu\right) B\left(V^{n} \mu\right)\right],
\end{aligned}
$$

where $n=0,1,2, \ldots$ and $k \in X$. Moreover, for the parameters $A\left(V^{n} \mu\right)$ and $B\left(V^{n} \mu\right)$, we obtain as follows:

$$
\begin{aligned}
& A\left(V^{n+1} \mu\right)=\sum_{p \in A_{1}}\left\{\begin{array}{l}
\left(1-r_{1}\right) r_{1}^{p}\left[A^{2}\left(V^{n} \mu\right)+B^{2}\left(V^{n} \mu\right)\right] \\
+\left(1-r_{2}\right) r_{2}^{p}\left[2 A\left(V^{n} \mu\right) B\left(V^{n} \mu\right)\right]
\end{array}\right\}, \text { and } \\
& B\left(V^{n+1} \mu\right)=\sum_{p \in A_{1}}\left\{\begin{array}{l}
{\left[1-\left(1-r_{1}\right) r_{1}^{p}\right]\left[A^{2}\left(V^{n} \mu\right)+B^{2}\left(V^{n} \mu\right)\right]} \\
+\left[1-\left(1-r_{2}\right) r_{2}^{p}\right]\left[2 A\left(V^{n} \mu\right) B\left(V^{n} \mu\right)\right]
\end{array}\right\} .
\end{aligned}
$$

It is obvious that the limit behaviour of the recurrent equation (17) depends on the limit behaviour of the recurrent equations (18).

Since $\quad A\left(V^{n} \mu\right)+B\left(V^{n} \mu\right)=1, \quad$ where $\quad A\left(V^{n} \mu\right) \geq 0 \quad$ and $B\left(V^{n} \mu\right) \geq 0$, then as $n \rightarrow \infty$ the recurrent equations (18) can be rewritten as follows:

$x=A\left(G_{r_{1}}\right)\left(x^{2}+y^{2}\right)+2 A\left(G_{r_{2}}\right)(x y)$, and

$y=B\left(G_{r_{1}}\right)\left(x^{2}+y^{2}\right)+2 B\left(G_{r_{2}}\right)(x y)$,

where $A\left(V^{n} \mu\right)$ and $B\left(V^{n} \mu\right)$ are represented as variable $x$ and $y$ respectively with $x \geq 0, y \geq 0$ and $x+y=1$.

It is sufficient to solve for the former equation in (19) which can be rewritten as follows:

$$
x=A\left(G_{r_{1}}\right)\left[x^{2}+(1-x)^{2}\right]+2 A\left(G_{r_{2}}\right)[x(1-x)] .
$$

It turns out that the equation (20) is the same quadratic equation as the equation (9). Since we are going to prove the regularity of this operator, we may apply the same procedure as applied in the case of Geometric quadratic stochastic operator generated by 2-partition $\xi$ of modulus 3 .

The main goal in investigating the regularity of quadratic stochastic operator is to show that the trajectory behaviour of such operator converges to a fixed point which indicates the existence of the limit of the sequence $V^{n} \mu(k)$.

In the previous section, it is proven that the equation (9) has a unique fixed point in the open interval $(0,1)$. It follows that any fixed point belongs to the open interval $(0,1)$ is attractive. Hence, Theorem 1 and Theorem 2 are employed for this particular case.

Therefore, the Geometric quadratic stochastic operator generated by 2-partition of arbitrary infinite points is a regular.

\section{CONCLUSION}

A Geometric quadratic stochastic operator generated by 2-partition $\xi$ of infinite points is a regular transformation.

\section{ACKNOWLEDGEMENT}

This research was financially supported by the International Islamic University Malaysia under the Research Initiative Grant Scheme RIGS16-311-0475.

\section{REFERENCES}

Akin, E., \& Losert, V. (1984). Evolutionary dynamics of zero-sum game. Journal of Mathematical Biology, 20, 231-258.

Akin, E. (1993). The General Topology of Dynamical Systems. USA: American Mathematical Society.

Bernstein, S. N. (1924). The solution of a mathematical problem related to the theory of heredity. Uchn Zapiski NI Kaf Ukr Otd Mat, 1, 83-115.

Ganikhodjaev, N. N., Ganikhodjaev, R. N., \& Jamilov, U. (2015). Quadratic stochastic operators and zero-sum game dynamics. Ergodic Theory and Dynamical Systems, 35(5), 1443-1473.

Ganikhodjaev, N., \& Hamzah, N. Z. A. (2014a). Nonhomogeneous Poisson 
nonlinear transformations on countable infinite set. 3rd International Conference on Mathematical Applications in Engineering (ICMAE' 14).

Ganikhodjaev, N., \& Hamzah, N. Z. A. (2014b). On Gaussian nonlinea transformations. Simposium Kebangsaan Sains Matematik Ke-22 (SKSM22).

Ganikhodjaev, N., \& Hamzah, N. Z. A. (2014c). On Poisson nonlinear transformations. The Scientific World Journal, 1-7.

Ganikhodjaev, N., \& Hamzah, N. Z. A. (2015a). Geometric quadratic stochastic operator on countable infinite set. AIP Conference Proceedings, 1643(1), 706-712.

Ganikhodjaev, N., \& Hamzah, N. Z. A. (2015b). Quadratic stochastic operators on segment $[0,1]$ and their limit behavior. Indian Journal of Science and Technology, 8(30).

Ganikhodjaev, N., \& Hamzah, N. Z. A. (2015c). On Volterra quadratic stochastic operators with continual state space. AIP Conference Proceedings, 1660, 050025.

Ganikhodjaev, R. N. (1993). Quadratic stochastic operators, Lyapunov functions, and tournaments. Russian Academy of Sciences. Sbornik Mathematics, 76, 489-506.

Ganikhodjaev, R. N. (1994). A chart of fixed points and Lyapunov functions for a class of discrete dynamical systems. Mathematical Notes, 56(5-6), 11251131.

Ganikhodzhaev, N. N., \& Zanin, D. V. (2004). On a necessary condition for the ergodicity of quadratic operators defined on the two-dimensional simplex. Russian Mathematical Surveys, 59(3), 161-162.

Ganikhodzhaev, R., Mukhamedov, F., \& Rozikov, U. (2011). Quadratic Stochastic Operators and Processes: Results and Open Problems. Infinite Dimensional Analysis, Quantum Probability and Related Topics, 14(02),
$279-335$.

Hofbauer, J., \& Sigmund, K. (1998). Evolutionary games and population dynamic. UK: Cambridge University Press.

Jenks, R. D. (1969). Quadratic differential systems for interactive population models. Journal of Differential Equations, 5, 497-514.

Karim, S. N., Hamzah, N. Z. A., \& Ganikhodjaev, N. (2019). A class of geometric quadratic stochastic operator on countable state space and its regularity. Malaysian Journal of Fundamental and Applied Sciences, 15(6), 872-877.

Kesten, H. (1970). Quadratic transformations: A model for population growth II. Advances in Applied Probability, 2, 179-229.

Losert, V. \& Akin, E. (1983). Dynamics of games and genes: Discrete versus continuous time. Journal of Mathematical Biology, 17(2), 241-251.

Lyubich, Y. I. (1978). Basic concepts and theorems of the evolution genetics of free populations. Russian Mathematical Surveys, 26, 51-123.

Lyubich, Y. I. (1992). Mathematical structures in population genetics. Berlin: Springer.

Mukhamedov, F., \& Embong, A. F. (2015). On b-bistochastic quadratic stochastic operators. Journal of Inequalities and Applications, 2015(1).

Mukhamedov, F. (2000). On infinite dimensional Volterra operators. Russian Mathematical Surveys, 55(6), 1161-1162.

Ulam, S. (1960). A Collection of Mathematical Problems. New York: Interscience.

Volterra, V. (1931). Variations and fluctuations of the number of individuals in animal species living together in animal ecology. New York: McGrawHill.

Zakharevich, M. I. (1978). On behavior of trajectories and the ergodic hypothesis for quadratic transformations of the simplex. Russian Mathematical Surveys, 33, 265-266. 